\title{
Bimodal distribution of the autocorrelation function in gamma-ray bursts
}

\author{
L. Borgonovo \\ Stockholm Observatory, 10691 Stockholm, Sweden \\ Received 23 October 2003 / Accepted 2 February 2004

\begin{abstract}
Autocorrelation functions (ACFs) are studied for a sample of 16 long gamma-ray bursts (GRBs) with known redshift $z$, that were observed by the BATSE and Konus experiments. When corrected for cosmic time dilation, the ACF shows a bimodal distribution. A narrow width class (11 bursts) has at half-maximum a mean width $\tau_{0}^{\prime}=1.6 \mathrm{~s}$ with a relative dispersion of $\sim 32 \%$, while a broad width class (5 bursts) has $\tau_{0}^{\prime}=7.5 \mathrm{~s}$ with a $\sim 4 \%$ dispersion. The separation between the two mean values is highly significant $(>7 \sigma)$. This temporal property could be used on the large existing database of GRBs with unknown redshift. The broad width set shows a very good linear correlation between width at half-maximum and $(1+z)$, with a correlation coefficient $R=0.995$ and a probability of chance alignment $<0.0004$. The potential application of this correlation to cosmology studies is discussed, using it in combination with recently proposed luminosity indicators.
\end{abstract}

Key words. gamma rays: bursts - gamma rays: observations - methods: data analysis - cosmology: distance scale

\section{Introduction}

The knowledge of time scales and source distances are essential for the physical understanding of astronomical phenomena. From the first detections in 1969 by Vela satellites (Klebesadel et al. 1973), until the launch of BeppoSAX in 1997, the distance scale of gamma-ray bursts (GRBs) remained unsettled. This mission provided arc-minute localization, leading to the discovery of a fading emission towards lower energy bands, the so-called afterglows. Thereafter, burst redshifts $z$ have been determined from spectroscopic analysis of the afterglows or, in some cases, of their associated host galaxies, proving that at least long-duration bursts are at cosmological distances. So far, the redshift of no short-duration burst has been clearly determined (although see Kulkarni et al. 2002). In this paper only the class of long GRBs will be considered (i.e., those with time duration $>2 \mathrm{~s}$ ).

To date, more than 30 burst redshifts have been spectroscopically measured thanks to immediate follow-up observations. On the other hand, there is a wealth of data from thousands of GRBs for which the redshift is unknown. Most of these were detected by the Burst and Transient Source Experiment (BATSE). Other important motivations to find a redshift estimator based only on the gamma-ray prompt emission are the lack of optical counterparts in some cases (the socalled dark afterglows), and the difficulty of spectroscopically determining redshifts beyond $z=5$ due to the Lyman alpha absorption. In recent years, two empirical relations have been

\footnotetext{
* e-mail: luis@astro.su.se
}

discovered to estimate the luminosity distance exclusively from the analysis of the gamma emission. One relates the isotropic luminosity to the time lag between different energy channels (Norris et al. 2000), and the other to a variability parameter of the light curve (Reichart et al. 2001). Both luminosity correlations can be used to derive luminosity distances and, assuming some specific cosmology, the corresponding redshifts. Thus, from these correlations it has been possible to estimate GRB luminosity functions and demographic distributions (see, e.g., Norris 2002; Lloyd-Ronning et al. 2002). These first estimations indicate that the GRB population may peak at redshift $z \sim 10$, being then ideal probes of the early universe. However, the luminosity functions derived in these works predict source counts $N(>P)$, as a function of photon flux $P$, that differ significantly from the observed one (Schmidt 2003). Much better calibration of these empirical relations is needed, and that will only be possible with a much larger number of independent redshift determinations covering a broader $z$ range.

Individual power density spectra (PDS) of GRB are in general very diverse, but the longest bursts show power-law spectra extended over two frequency decades. Shorter bursts also display this property by averaging the PDSs of a large sample (Beloborodov et al. 1998, 2000). This underlying powerlaw behavior indicates the absence of any preferred time scale. The autocorrelation function (ACF) is the Fourier transform of the PDS, therefore it contains in principle the same information that can be visualized in a different way. The ACF gives a measure of the correlation between different points in the light curve that are separated by a given time lag. Various efforts have been made using these data analysis tools to find a 
temporal characteristic that might correlate with the redshift and, e.g., Chang et al. (2002) have found a weak correlation between the power-law index of the PDS and $z$. See also Atteia (2003) for a proposed redshift indicator.

In this paper it will be shown that the ACF can be used to define characteristic times that strongly correlate with the redshift. In Sect. 2 the data selection and the use of the ACF are described. In Sect. 3 it is shown that the ACF corrected for time dilation effects has a bimodal distribution, and that this property could be used to construct an empirical relation to estimate $z$. Finally, the results and their possible applications are discussed in Sect. 4.

\section{Data and methods}

This work is mainly based on data taken by BATSE on board the Compton Gamma-Ray Observatory (CGRO; Fishman et al. 1989). It consisted of eight modules placed on each corner of the satellite, giving full sky coverage. Each module had two types of detectors: the Large Area Detector (LAD) and the Spectroscopy Detector (SD). The former had a larger collecting area and from it the CGRO Science Support Center (GROSSC) provided the so-called concatenated $64 \mathrm{~ms}$ burst data, which is a concatenation of the three standard BATSE data types DISCLA, PREB, and DISCSC. All three data types have four energy channels (approximately 25-55, 55-110, $110-320$, and $>320 \mathrm{keV}$ ). The DISCLA data is a continuous stream of $1.024 \mathrm{~s}$ and the PREB data covers the $2.048 \mathrm{~s}$ prior to the trigger time at $64 \mathrm{~ms}$ resolution, both types obtained from the 8 LADs. They have been scaled to overlap the DISCSC $64 \mathrm{~ms}$ burst data, that was gathered by the triggered LADs (usually the four closer to the line of sight). This combined data format was used when available, since the concatenated pre-burst data allows a better estimation of the background.

All BATSE bursts with known redshift $z$ were considered for study ${ }^{1}$. In some cases, like GRB 980326 and GRB 980613 , the data are incomplete or were not recorded. For burst GRB 970828 the DISCSC data are incomplete; but it was possible to derive data with the same characteristics from the MER data type, binning up the 16 energy channel into 4 DISCSC-like energy channels. For GRB 000131 the given DISCSC data are unevenly sampled and it had to be uniformly binned into $1.024 \mathrm{~s}$ time resolution. The BATSE sample total 11 cases.

To improve statistics, we also considered GRB data that are publicly available from other experiment. The Konus mission (Aptekar et al. 1995) publishes GRB light curves of $64 \mathrm{~ms}$ resolution within an energy band of 50-200 keV. At the time of this publication, there were 25 Konus bursts with known redshift. But the collecting area of this experiment is about 20 times smaller than the one on BATSE and consequently, in most cases, the signal is too weak for the purposes of this analysis. A total of 5 bursts were selected for this study.

\footnotetext{
${ }^{1}$ See J. Greiner web page for a compilation of all GRBs with known redshift at http://www.mpe.mpg.de/ jcg/grbgen.html
}

The INTEGRAL mission (launched in October 2002) makes public all count time histories of the bursts detected by the anti-coincidence shield of its gamma-ray spectrometer (SPI-ACS). It has a time resolution of $50 \mathrm{~ms}$ and a non-sharp lower energy threshold at about $80 \mathrm{keV}$ (Kienlin et al. 2001). So far, the only detected burst with known $z$ is GRB 030329, and it was also detected by Konus. Therefore, these data were used here mainly for comparative purposes.

The autocorrelation function of GRBs was first studied by Link et al. (1993) and later on by, e.g., Fenimore et al. (1995) and Beloborodov et al. (2000). Following their notation, from a uniformly sampled count history with $\Delta T$ time resolution and $N$ time bins, let $m_{i}$ be the total observed counts at bin $i$. Also let $b_{i}$ be the corresponding background level and $c_{i}=m_{i}-b_{i}$ the net counts. The discrete ACF as a function of the time lag $\tau=k \Delta T$ is

$A(\tau)=\sum_{i=0}^{N-1} \frac{c_{i} c_{i+k}}{A_{0}}, \quad k=1, \ldots, N-1$

and $A(0)=1$ for $k=0$. Here the periodic boundary conditions $\left(c_{i}=c_{i+N}\right)$ are assumed. The normalization constant $A_{0}$ is defined as

$A_{0}=\sum_{i=0}^{N-1} c_{i}^{2}-m_{i}$

The normalization makes the ACF of each burst fluenceindependent. The term $m_{i}$ in Eq. (2) subtracts the contribution of the uncorrelated noise assuming that it follows Poisson statistics. This is not the case for the pre-trigger data of the concatenated BATSE data type and they were excluded after the background fitting. Their contribution to the ACF is negligible but they would affect the estimation of $A_{0}$. This should also be considered when doing power density spectral analysis. For practical reasons, the actual calculation of Eq. (1) was done using a Fast Fourier Transform (FFT) routine, i.e., squaring the absolute value of the transformed gives the power density spectrum, and taking the inverse transform of it gives the ACF. Zero padding of the time series was used to avoid the artifacts produced by the periodic boundary condition.

The background estimation was done by fitting with up to a second order polynomial the pre- and post-burst data, that was judged by visual inspection to be inactive. This is particularly critical for weak bursts. Unfortunately, the Konus GRB light curves that are publicly available have a fixed duration of $100 \mathrm{~s}$, with no pre-burst data, and sometimes not even postburst data. Only a few cases are sufficiently bright and have long post-burst data to allow a reliable estimation of the ACF. The problem was studied using numerical simulations and it became clear that for most of the Konus set the systematic errors introduced by the background estimation are the main source of uncertainty. Figure 1 shows comparisons of the ACFs of bursts for which there are data from both the Konus and BATSE experiments. As reported by Fenimore et al. (1995), the ACF of GRBs narrows at higher energies. The best match was obtained using the sum of the BATSE energy channels 2 and 3, covering a similar energy range as the corresponding Konus data. Note that the agreement will depend mainly on having a 

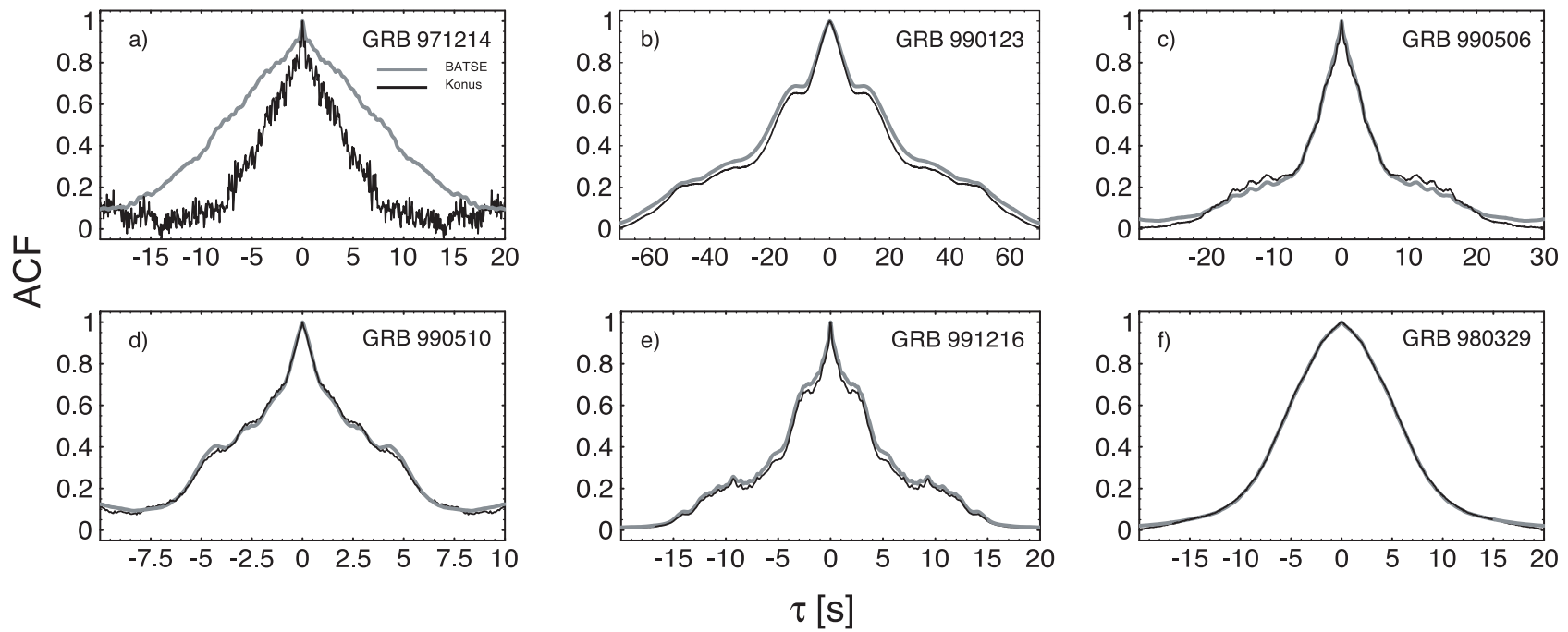

Fig. 1. Comparison of the ACFs of 6 GRBs obtained using data from two different experiments. Solid lines: Konus 64 ms data in the $50-200 \mathrm{keV}$ energy band; gray lines: BATSE $64 \mathrm{~ms}$ data in the 55-320 keV energy range. There is sufficiently good agreement for the bright bursts, when the results are not very sensitive to the background estimation. GRB 971214 is considerably dimmer than the others (see the text for discussion).

similar lower-end energy limit, since there are more counts at lower energies and the ACF is a quadratic function of the number of counts. The Konus weak case GRB 971214 illustrates how a poor estimation of the background affects the ACF calculation. On the other hand in the strong case GRB 990123, even with a short post-burst data tail to fit the background, the difference between the ACFs is acceptable for the purposes of this work. Guided by this comparison, the selection criteria for the Konus cases were set, requiring peak count rates larger than 3000 counts $\mathrm{s}^{-1}$ and post-burst data. These criteria are met by all bursts shown in Fig. 1 except the first, and by 5 other cases not observed by BATSE that were then added to the sample. Among these last cases is the bright GRB 030329 that was also observed by INTEGRAL, and Fig. 2 shows the good agreement between the ACFs derived using the two different instrument data. Table 1 summarizes in its four first columns the adopted sample of GRBs, the instrument source, the estimated redshift $z$ and the corresponding reference.

\section{Results}

In Fig. 3a the ACFs of all the GRB sample are shown. One can see that at different heights the width of the ACFs has a fairly uniform distribution, with the half-width $\tau_{0}$ ranging, e.g., at half-maximum between $2.5-20 \mathrm{~s}$. Figure $3 \mathrm{~b}$ shows the local autocorrelation function $A\left(\tau^{\prime}\right)$, where the cosmic time dilation has been removed, and $\tau^{\prime}=\tau /(1+z)$ is the corrected time lag. The width of the different ACFs shows now, particularly around the half-maximum level, a bimodal distribution with a clear gap between two sets: a broad width set of 5 bursts and a narrow width set of 11 bursts. The redshift of GRB 980329 is only known to be in the range $z=2.0-3.9$ (Lamb et al. 1999). Thus, for Fig. $3 \mathrm{~b}$ an average value $z=3$ was chosen, but in any case for the given $z$ range its ACF will lie within the other narrow width bursts. This burst was used here to show the bimodality but will be excluded from the following calculations.

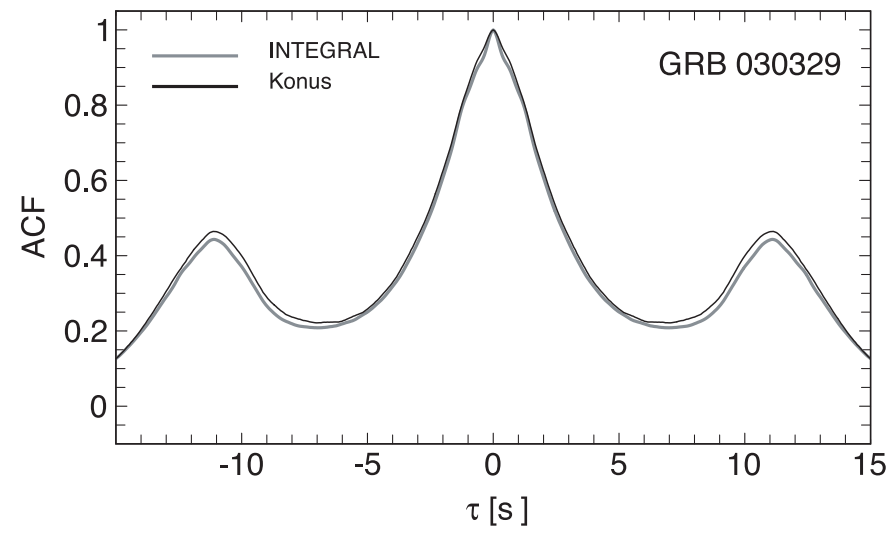

Fig. 2. Two ACF functions of GRB 030329. Solid line: Konus $64 \mathrm{~ms}$ data in the 50-200 keV energy band; gray line: INTEGRAL $50 \mathrm{~ms}$ data with a soft low energy cut-off at $\sim 80 \mathrm{keV}$. The high secondary peak of the ACF reflects the two bright pulse structure of the light curve.

For the 11 BATSE bursts the local ACF distribution was analyzed at different energy channels. Although narrower at larger energies, the ACF shows the same clear bimodal distribution in all channels.

To study the distribution of the local ACF, e.g., to estimate statistical moments at different lags, one cannot simply add the time series since now all of them have time bins of different sizes. To overcome this problem, the logarithm of each discrete ACF was approximated by a polynomial function $f(\tau) \simeq \ln A(\tau)$. A high degree polynomial was used (typically $\sim 12$ ) to match the data within the range of the random fluctuations up to time lags of 10 and $30 \mathrm{~s}$ for narrow and broad cases respectively. These ranges were chosen to well cover the central peaks of the ACFs down to the 0.1 level. Using these functions, the mean and the sample standard deviation $s$ were 

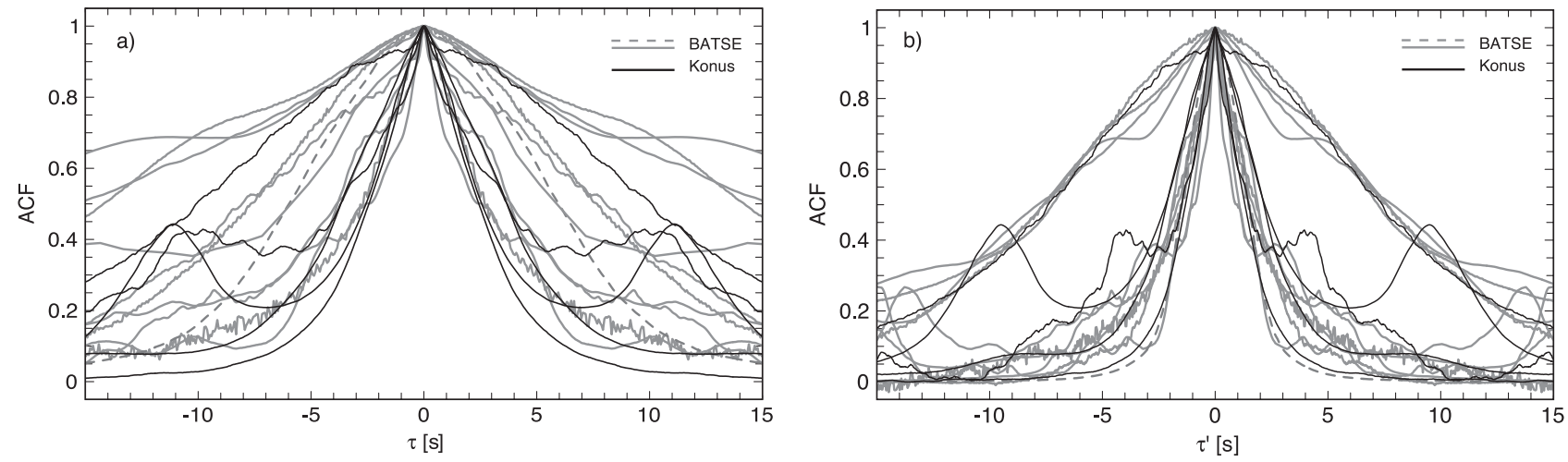

Fig. 3. a) Autocorrelation functions of the 16 bursts sample. BATSE and Konus cases are shown in gray and solid lines respectively. b) Local ACFs, where time dilation due to cosmic effect has been corrected, being $\tau^{\prime}=\tau /(1+z)$. GRB 980329 is shown with dashed lines assuming a redshift $z=3$.

Table 1. Sample of 16 GRBs with known redshift. The 6 columns give the name of the GRB, the instrument, the measured redshift $z$, the corresponding reference, the ACF half-width at half-maximum $\tau_{0}$, and the width corrected for time dilation $\tau_{0}^{\prime}$.

\begin{tabular}{llllll}
\hline \hline GRB & Instrument & $z$ & Ref. & $\tau_{0}(s)$ & $\tau_{0}^{\prime}(s)$ \\
\hline 970508 & BATSE & 0.835 & 1 & 2.70 & 1.47 \\
970828 & BATSE & 0.9578 & 2 & 15.33 & 7.83 \\
971214 & BATSE/Konus & 3.418 & 3 & 8.02 & 1.81 \\
980329 & BATSE/Konus & $3 \pm 1$ & 4 & 5.96 & 1.49 \\
980425 & BATSE & 0.0085 & 5 & 7.62 & 7.56 \\
980703 & BATSE & 0.966 & 6 & 14.15 & 7.19 \\
990123 & BATSE/Konus & 1.600 & 7 & 19.81 & 7.62 \\
990506 & BATSE/Konus & 1.3066 & 8 & 3.83 & 1.66 \\
990510 & BATSE/Konus & 1.619 & 9 & 2.54 & 0.97 \\
991208 & Konus & 0.7055 & 10 & 3.67 & 2.15 \\
991216 & BATSE/Konus & 1.02 & 11 & 3.80 & 1.88 \\
000131 & BATSE & 4.500 & 12 & 5.77 & 1.05 \\
000210 & Konus & 0.846 & 13 & 2.43 & 1.31 \\
010222 & Konus & 1.477 & 14 & 3.68 & 1.48 \\
011121 & Konus & 0.362 & 15 & 9.98 & 7.32 \\
030329 & Konus/INTEGRAL & 0.1685 & 16 & 2.56 & 2.19 \\
\hline
\end{tabular}

1 Metzger et al. (1997).

2 Djorgovski et al. (2001).

3 Kulkarni et al. (1998).

4 Lamb et al. (1999).

5 Tinney et al. (1998).

6 Djorgovski et al. (1998).

7 Kulkarni et al. (1999).

8 Bloom et al. (2003).

9 Beuermann et al. (1999).

10 Dodonov et al. (1999).

11 Vreeswijk et al. (1999).

12 Andersen et al. (2000).

13 Piro et al. (2002).

14 Jha et al. (2001).

15 Garnavich et al. (2003).

16 Greiner et al. (2003).

calculated for the two sets. Since the sample size $n$ is small in both cases, the standard deviation $\sigma$ was estimated as

$\sigma=s\left(1+\frac{1}{\sqrt{2(n-1)}}\right)$,

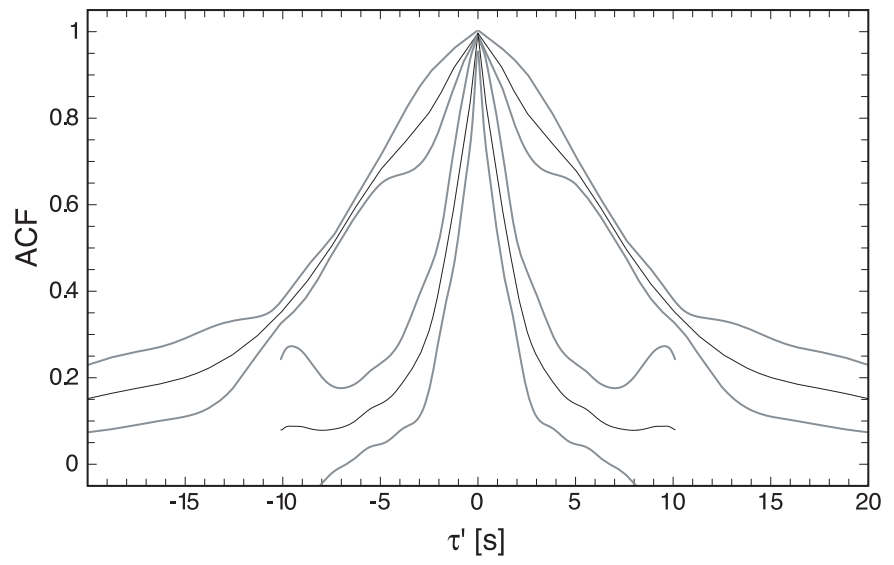

Fig. 4. Mean value of the local ACF for narrow and broad width bursts (solid lines). The $1 \sigma$ region about the mean is also shown (gray lines). At half-maximum $A\left(\tau^{\prime}\right)=0.5, \tau^{\prime}=(1.6 \pm 0.5) \mathrm{s}$ and $\tau^{\prime}=(7.5 \pm 0.3) \mathrm{s}$ for narrow and broad widths respectively.

using a correction for low number statistics assuming normal distributions. Figure 4 shows the mean curves and the $\pm 1 \sigma$ region around them. At half-maximum $A\left(\tau_{0}^{\prime}\right)=0.5$, the halfwidth is $\tau_{0}^{\prime}=(1.6 \pm 0.5) \mathrm{s}$ and $\tau_{0}^{\prime}=(7.5 \pm 0.3) \mathrm{s}$ for narrow and broad width sets respectively. Hence, at half-maximum the distributions do not overlap even at the $7 \sigma$ level. The probability of such separation into two sets by chance, having an underlying unimodal distribution, was estimated numerically. The overall distribution was assumed uniform in a given range (most favorable case). Considering the same total number of points (15), and asking conservatively the size of the smallest set to be $\geq 4$, the probability $p$ of obtaining two sets with a difference between their means $>7 \sigma$ is $p<6 \times 10^{-7}$. Therefore, the alternative hypothesis of having a bimodal distribution can be accepted at a highly significant confidence level.

The small range of the broad width distribution around the half-maximum level is particularly interesting, because it represents a relative dispersion of $4 \%$, while the relative dispersion of the narrow width is $32 \%$. This means that if we had a way to know a priori at what width class a burst belongs, we would 


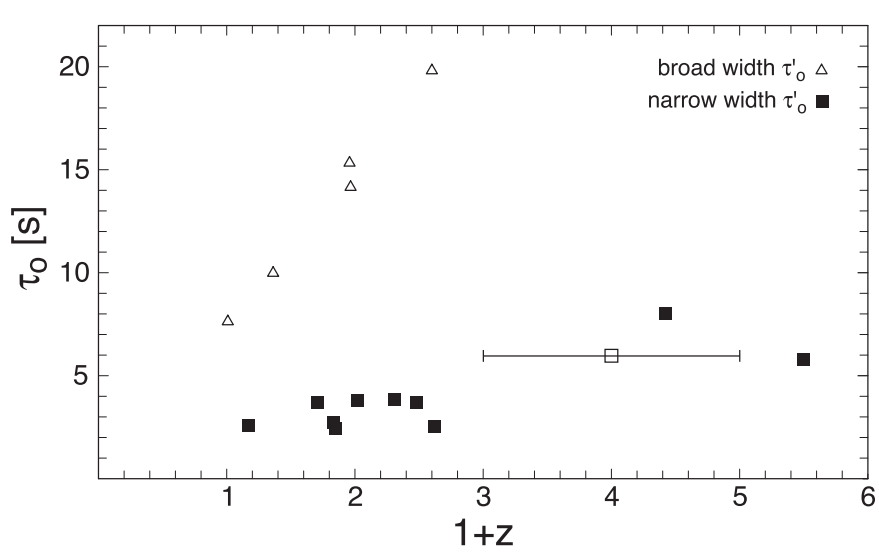

Fig. 5. Correlation between the width at half-maximum $\tau_{0}$ and the dilation factor $1+z$. Bursts are classified into narrow and broad width $\tau_{0}^{\prime}$ cases, as seen by a local observer, and they are marked by squares and triangles respectively. GRB 980329 is indicated with a hollow square; its redshift is only known to be within the shown $z$ range. A linear fit of the form $f(x)=a x$ gives $a_{n}=(1.42 \pm 0.15) \mathrm{s}$ and $a_{b}=(7.54 \pm 0.10) \mathrm{s}$ for narrow and broad sets. The slopes are approximately equal to the average $\tau_{0}^{\prime}$ for each set. Note that since the slope ratio is 5.3 , for a given $\tau_{0}$ each correlation predicts very different $1+z$ values.

be able to estimate with the same corresponding uncertainties the time dilation factor $1+z$, and therefore the redshift in practice only when $z \gtrsim 1$. The width $\tau_{0}$ was calculated fitting the logarithm of the ACF in the range $0.4 \leq A(\tau) \leq 0.6$ with a second degree polynomial. In the last two columns of Table 1 , the obtained values for $\tau_{0}$ and $\tau_{0}^{\prime}=\tau_{0} /(1+z)$ are listed. Figure 5 shows $\tau_{0}$ versus $1+z$ for both width classes. As expected, the broad width set shows a very good correlation, with a linear correlation coefficient $R=0.995$ and a probability of chance alignment $p<0.0004$. The corresponding values for the narrow width set are $R=0.809$ and $p<0.005$ respectively. Notice that GRB 980425, which has been associated with SN 1998bw, belongs to the broad width set. This burst was considered an outlier in the studies of the lag and variability luminosity correlations when modeling the data with a single power-law, although its inclusion supports the general trend in both cases.

\section{Discussion and conclusions}

The average PDS of bursts shows an overall power-law behavior, indicative of a self-similar underlying process where there are no preferred timescales. If this is the case, then the width of the ACF is related to the low-frequency cut-off of the PDS, which is due to the finite duration of the burst (Beloborodov et al. 2000). As mentioned in Sect. 1, in principle the information given by the ACF and the PDS is the same. In practice, since they express this information differently, they are affected by noise and window effects in different ways. It would be difficult to make a good estimation of the low-frequency cut-off in the PDS due to the large statistical fluctuations. On the other hand, the width at half-maximum of the ACF gives a robust measure.

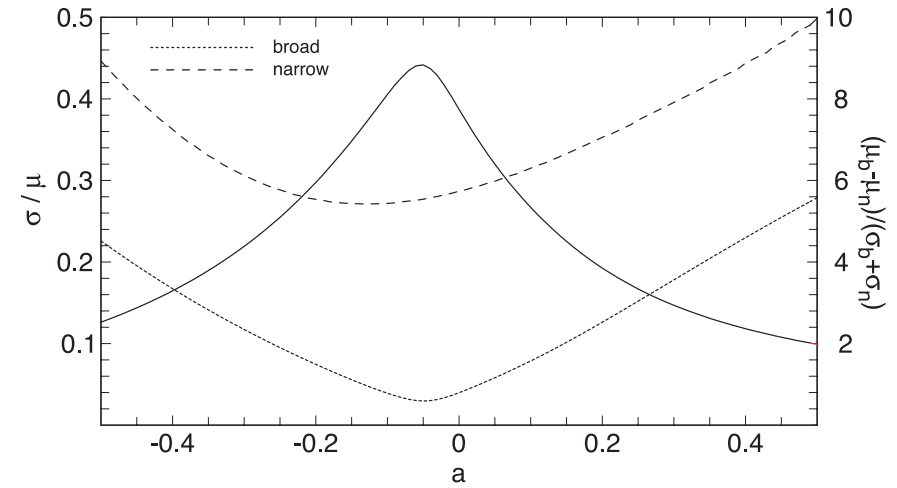

Fig. 6. Variation of the dispersion of the ACF width with additional redshift dependencies. The local values are calculated assuming $\tau_{0}^{\prime}=$ $\tau_{0} /(1+z)^{1+a}$, where $a$ is an unknown correction index. The left ordinate shows the relative dispersion of the broad (dotted line) and narrow (dashed line) sets. Dispersion minima occur at $a \ll 1$ in both cases. The right ordinate shows for the solid line the difference between the mean values of each set $\left(\mu_{b}, \mu_{n}\right)$ in number of total standard deviations $\left(\sigma_{b}+\sigma_{n}\right)$. The gap between sets remains above $3 \sigma$ over a large range $(-0.4<a<0.3)$, indicating the robustness of the bimodality result to additional corrections.

In this analysis the ACFs of bursts were only corrected for the cosmic time dilation. However, since the detectors are sensitive over a finite energy band, effects due to the shift in energy should also be present. Studying a set of 45 bright long bursts, Fenimore et al. (1995) found that the full-width $W$ of the average $\mathrm{ACF}$ (at the $e^{0.5}$ level) depends on the energy $E$ as $W(E) \propto E^{-0.4}$. This narrowing of the ACF should partially counteract the time stretching since for large redshifts the energy window of the instrument will see photons emitted at higher mean energies. Furthermore, due to the trigger threshold, bursts detected at high redshifts are more luminous. There are indications that the pulse width, and therefore the ACF width, correlates with the luminosity (Lee et al. 2000). One should consider also that because of the energy shift, bursts at high redshifts are detected at earlier stages. If the local ACF has an approximately constant width $\tau_{0}^{\prime}$ (for each width class) these effects should produce a deviation from linearity in Fig. 5. Since no important deviation is observed, the net combined effect must be small. To explore how sensitive our results are to such effects we will assume now that the local width of the ACF is given by $\tau_{0}^{\prime}=\tau_{0} /(1+z)^{1+a}$, where the index $a$ takes into account additional redshift dependencies. Figure 6 shows the relative dispersion of the width $\tau_{0}^{\prime}$ for each set as a function of $a$. The dispersion minima occur at small $a$ index values in both cases, with $a_{\text {min }}^{(b)}=-0.05 \pm 0.05$ and $a_{\text {min }}^{(n)}=-0.13 \pm 0.23$ for the broad and narrow width sets respectively. The difference between the mean values of each set versus $a$ is also shown in Fig. 6. It peaks at $a=-0.05$ with $8.8 \sigma$, where now $\sigma$ is the total standard deviation. The gap between sets remains larger than $3 \sigma$ over a large range $(-0.4<a<0.3)$, indicating how robust the bimodality result is to any additional correction.

The practical use of the proposed empirical relations requires a criterion to decide to what width class a burst belongs. 


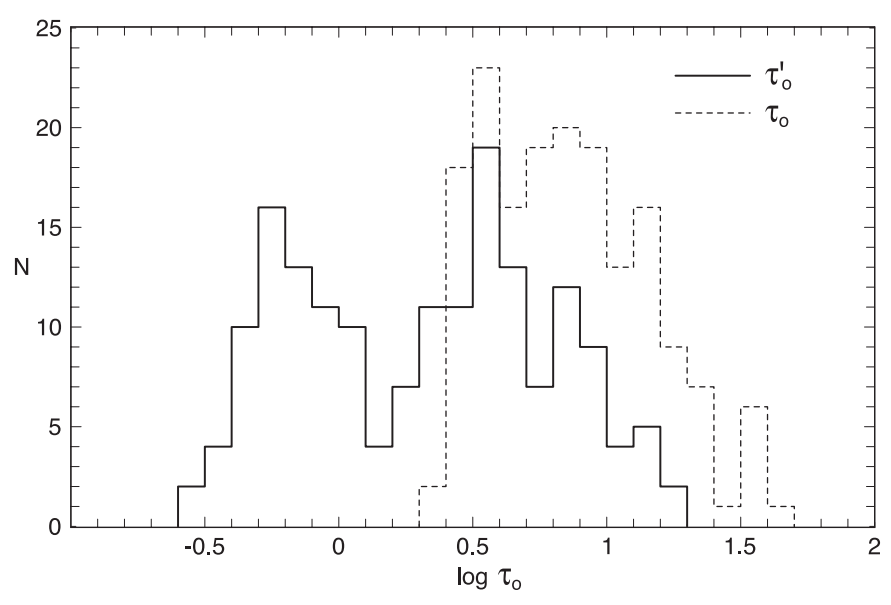

Fig. 7. Distribution of the ACF width at half-maximum for a sample of 170 BATSE GRBs. Dashed-line: logarithmic histogram of the width $\tau_{0}$ (in seconds). The distribution seems unimodal. Solid-line: The width corrected for cosmic time dilation $\tau_{0}^{\prime}$, using estimated redshifts derived from the luminosity-variability correlation (Fenimore \& Ramirez-Ruiz 2000). The distribution appears now bimodal, with median values approximately $0.7 \mathrm{~s}$ and $4.0 \mathrm{~s}$ for each subset.

For a width $\tau_{0} \lesssim 7.5 \mathrm{~s}$ the two classes do not overlap (see Fig. 5). On the other hand, if extrapolations are valid, $\tau_{0} \gg 20 \mathrm{~s}$ implies for the narrow width class unrealistically large $z$. No burst spectral characteristic was found to correlate with the width class. The same was true for the luminosity and total energy release, but again larger samples should be studied to be conclusive. The mentioned luminosity correlations could give a first $z$ estimation to determine the width class, and then it will be possible using the ACF to obtain a second better and independent estimation. Since the two classes are separated by more than a factor 5, the selection should not depend in practice on the assumed cosmological parameters, and therefore the ACF width-redshift correlation could be used in addition to constrain them.

To investigate the combined use of these correlations, the ACFs of a BATSE burst sample studied by Fenimore \& Ramirez-Ruiz (2000) were calculated. They estimated the redshifts of 220 GRBs using a power-law fit of the luminosity $L$ versus the variability $V$ based on only 7 bursts. Therefore they obtained the best-fit parameters for their model $L \propto V^{\alpha}$ with large uncertainties. Furthermore, the best-fit parameters vary considerably if GRB 980425 is included in the calibration, and this burst was excluded in the derivation of the published redshifts. Figure 7 shows the logarithmic distribution of the observed ACF widths $\tau_{0}$ derived for this sample (dashedline) and the distribution of the widths $\tau_{0}^{\prime}$ corrected for time dilation (solid-line). In Fig. 7 those cases where the iterative method to determine $z$ diverged have been excluded, as well as bursts with observed ACF widths $\tau_{0}<2.4 \mathrm{~s}$, but our conclusions do not depend on this selection. While the uncorrected distribution seems unimodal, the corrected one appears bimodal, in qualitative agreement with the results of Sect. 3. The probability $p$ that a statistical fluctuation could produce this feature was estimated. Assuming an underlying log-normal distribution, a conservative estimation gives $p<0.02$. The $\tau_{0}^{\prime}$ distribution is broad, but that is to be expected given the large spread found in the luminosity-variability correlation. The median values for the two width subsets are $\sim 0.7 \mathrm{~s}$ and $\sim 4.0 \mathrm{~s}$, both approximately a factor 2 smaller than the mean values of the distributions shown in Fig. 4. However, this discrepancy can be accounted for considering the uncertainties. Based on the analysis of 20 bursts with known redshifts Reichart et al. (2001) estimated $\alpha=3.3_{-0.9}^{+1.1}$. In particular, the exclusion of GRB 980425 will overestimate the exponent $\alpha$ and consequently the redshifts, given smaller $\tau_{0}^{\prime}$ corrected widths.

The ACF width-redshift correlations described here will need to be confirmed by a larger statistical sample. Additionally, the lag and variability luminosity correlations need to be known for a larger redshift range to avoid uncertain extrapolations. The close agreement between the ACFs using data from the past mission BATSE and the presently operating Konus and INTEGRAL will allow us to continue improve the statistic of this work. In combination with the luminosity correlations we should be able to construct a GRB-based Hubble diagram (i.e., a luminosity distance versus $z$ plot) for high $z$, following a procedure similar to that of Schaefer (2003). Such a diagram would have important implications in cosmology studies. Ongoing efforts in this direction will be presented in the near future.

Acknowledgements. I wish to thank S. Larsson, C.-I. Björnsson, and F. Ryde for useful comments and careful reading of the manuscript. This research has made use of BATSE and Konus data obtained from the High Energy Astrophysics Science Archive Research Center (HEASARC), provided by NASA's Goddard Space Flight Center.

\section{References}

Andersen, M. I., Hjorth, J., Pedersen, H., et al. 2000, A\&A, 364, L54 Aptekar, R. L., Frederiks, D. D., Golenetskii, S. V., et al. 1995, Space Sci. Rev., 71, 265

Atteia, J.-L. 2003, A\&A, 407, L1

Beloborodov, A. M., Stern, B. E., \& Svensson, R. 1998, ApJ, 508, L25 Beloborodov, A. M., Stern, B. E., \& Svensson, R. 2000, ApJ, 535, 158

Beuermann, K., Hessman, F. V., Reinsch, K., et al. 1999, A\&A, 352, L26

Bloom, J. S., Berger, E., Kulkarni, S. R., Djorgovski, S. G., \& Frail, D. A. 2003, AJ, 125, 999

Chang, H.-Y., Yoon, S.-J., \& Choi, C.-S. 2002, A\&A, 383, L1

Djorgovski, S. G., Kulkarni, S. R., Bloom, J. S., et al. 1998, ApJ, 508, L17

Djorgovski, S. G., Frail, D. A., Kulkarni, S. R., et al. 2001, ApJ, 562, 654

Dodonov, S. N., Afanasiev, V. L., Sokolov, V. V., Moiseev, A. V., \& Castro-Tirado, A. J. 1999, GCN Report, 475

Fenimore, E. E., \& Ramirez-Ruiz, E. 2000, [arXiv:astro-ph/0004176]

Fenimore, E. E., int't Zand, J. J. M., Norris, J. P., Bonnell, J. T., \& Nemiroff, R. J. 1995, ApJ, 448, L101

Fishman, G. J., et al. 1989, in Proc. of the GRO Science Workshop, ed. W. N. Johnson, 2

Garnavich, P. M., Stanck, K. Z., Wyrzykowski, L., et al. 2003, ApJ, 582,924 
Greiner, J., Peimbert, M., Estaban, C., et al. 2003, GCN Report, 2020 Jha, S., Pahre, M. A., Garnavich, P. M., et al. 2001, ApJ, 554, L155 von Kienlin, A., Arend, N., \& Lichti, G. G. 2001, Gamma-ray Bursts in the Afterglow Era, ed. E. Costa, F. Frontera, \& J. Hjorth (Berlin, Heidelberg: Springer), 427

Klebesadel, R. W., Strong, I. B., \& Olson, R. A. 1973, ApJ, 182, L85

Kulkarni, S. R., Djorgovski, S. G., Ramaprakash, A. N., et al. 1998, Nature, 393, 35

Kulkarni, S. R., Djorgovski, S. G., Odewahn, S. C., et al. 1999, Nature, 398, 389

Kulkarni, S. R., Goodrich, R., Berger, E., et al. 2002, GCN Report, 1428, 1

Lamb, D. Q., Castander, F. J., \& Reichart, D. E. 1999, A\&AS, 138, 479

Lee, A., Bloom, E. D., \& Petrosian, V. 2000, ApJS, 131, 21

Link, B., Epstein, R. I., \& Priedhorsky, W. C. 1993, ApJ, 408, L81
Lloyd-Ronning, N. M., Fryer, C. L., \& Ramirez-Ruiz, E. 2002, ApJ, 574,554

Metzger, M. R., Djorgovski, S. G., Kulkarni, S. R., et al. 1997, Nature, 387,878

Norris, J. P. 2002, ApJ, 579, 386

Norris, J. P., Marani, G. F., \& Bonnell, J. T. 2000, ApJ, 534, 248

Piro, L., Frail, D. A., Gorosakel, J., et al. 2002, ApJ, 577, 680

Reichart, D. E., Lamb, D. Q., Fenimore, E. E., et al. 2001, ApJ, 552, 57

Schaefer, B. E. 2003, ApJ, 583, L67

Schmidt, M. 2003 [arXiv: astro-ph/0301277]

Tinney, C., Stathakis, R., Cannon, R., et al. 1998, IAU Circ., 6896

Vreeswijk, P. M., Rol, E., Hjorth, J., et al. 1999, GCN Report, 496

Weisskopf, M. C., Kahn, S. M., \& Sutherland, P. G. 1975, ApJ, 199, L147 